\title{
Danon disease presenting with dilated cardiomyopathy and a complex phenotype
}

\author{
Matthew R. G. Taylor $\cdot$ Lisa Ku $\cdot$ Dobromir Slavov $\cdot$ Jean Cavanaugh $\cdot$ Mark Boucek \\ Xiao Zhu · Sharon Graw · Elisa Carniel · Carl Barnes · Dianna Quan · Ryan Prall · \\ Mark A. Lovell · Gary Mierau · Patsy Ruegg • Naresh Mandava · Michael R. Bristow • \\ Jeffrey A. Towbin - Luisa Mestroni · on behalf of the Familial Cardiomyopathy Registry
}

Received: 13 March 2007 / Accepted: 28 July 2007/Published online: 26 September 2007

(C) The Japan Society of Human Genetics and Springer 2007

\begin{abstract}
X-linked dilated cardiomyopathy (XLCM) was first described in 1987 and associated with dystrophin gene $(D M D)$ mutations a decade later in one of the original two families. Here we report long-term follow-up of the second family (XLCM-2), for which a $D M D$ mutation was never found. Analysis of the lysosome-associated membrane protein-2 (LAMP-2) gene detected a novel mutation, confirming a diagnosis of Danon disease. The broad phenotype in this family included dilated and hypertrophic cardiomyopathy, cardiac pre-excitation, skeletal myopathy with high serum creatine kinase, cognitive impairment (in males), and a pigmentary retinopathy in affected females. Cardiac biopsy specimens showed extensive vacuolar changes in an affected adult male. Remarkably, the skeletal muscle biopsy in a 13-month-old mutation-carrying male showed no vacuolization by standard histology. We conclude that XLCM may be the presenting sign of Danon
\end{abstract}

Supported by American Heart Association (0150453N), Muscular Dystrophy Association (PN0007-056) and NIH (1RO1HL69071-01 and 1K23H167915-01A1).

M. R. G. Taylor $\cdot$ L. Ku - D. Slavov $\cdot$ X. Zhu $\cdot$ S. Graw

E. Carniel - C. Barnes - D. Quan · R. Prall · P. Ruegg ·

N. Mandava $\cdot$ M. R. Bristow $\cdot$ L. Mestroni

University of Colorado at Denver and Health Sciences Center,

Aurora, CO, USA

J. Cavanaugh · M. Boucek · M. A. Lovell - G. Mierau

The Children's Hospital, Denver, CO, USA

J. A. Towbin

Baylor College of Medicine, Houston, TX, USA

M. R. G. Taylor $(\bowtie)$

12635 East Montview Blvd. Suite 100, Aurora,

CO 80045, USA

e-mail: matthew.taylor@uchsc.edu disease and, in the presence of a familial history of HCM, pre-excitation, skeletal muscle involvement and retinal pigmentary dystrophy should prompt LAMP-2 clinical testing. Furthermore, the absence of vacuolar myopathy in biopsies from young patients may not exclude Danon disease.

Keywords Danon disease - LAMP-2 .

Dilated cardiomyopathy · Wolff-Parkinson-White

\section{Introduction}

X-linked dilated cardiomypathy (XLCM) was first described by Berko and Swift in a large family characterized by cardiomyopathy and high serum creatine-kinase (CK) in males, and slow progression of the disease in females (Berko and Swift 1987). Linkage to the X-chromosome was shown in this family and a second family, XLCM-2 (Towbin et al. 1993). A dystrophin gene (DMD) mutation was found in the larger family; a $D M D$ mutation was not detected in XLCM-2, which consisted of a mildly affected mother who had three sons manifesting a dilated cardiomyopathy phenotype (Ortiz-Lopez et al. 1997) Mutations in another X-linked gene, lysosome-associated membrane protein-2 (LAMP-2), are an increasingly recognized cause of X-linked cardiomyopathy. Also known as Danon disease, the original phenotype description included a triad of non-obstructive hypertrophic cardiomyopathy (HCM), skeletal myopathy, and mental retardation in males (Danon et al. 1981). Histological skeletal muscle findings mimic those of Pompe disease, and the cardiomyopathy is most commonly described as hypertrophic in males. However, the phenotypic spectrum of Danon is still incompletely understood, and the role of dilated cardiomyopathy (DCM) 
Table 1 Clinical phenotypes of LAMP-2 mutation carriers

\begin{tabular}{|c|c|c|c|c|c|c|c|c|c|c|c|c|c|c|c|}
\hline 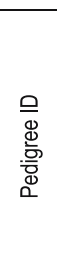 & 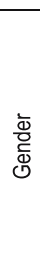 & 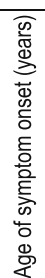 & 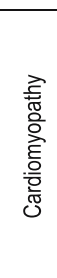 & 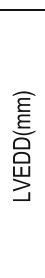 & 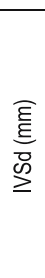 & $\frac{\widehat{\varrho}}{\frac{\mathrm{g}}{4}}$ & 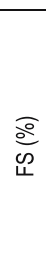 & 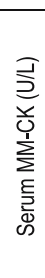 & 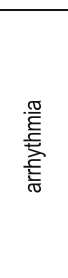 & 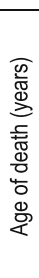 & 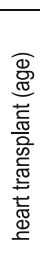 & 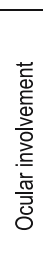 & 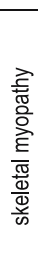 & 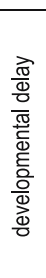 & 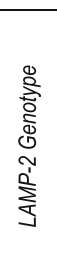 \\
\hline$\|: 3$ & $\mathrm{~F}$ & 58 & $\overline{D C M}$ & 64 & 12 & 29 & 19 & $(\mathrm{~N})$ & $\overline{\mathrm{AF}}$ & & $\mathrm{N}$ & & $\mathrm{N}$ & $\mathrm{N}$ & $\mathrm{M} / \mathrm{wt}$ \\
\hline III:1 & $F$ & 38 & $\mathrm{~N}$ & 48 & 11 & 64 & 26 & $(\mathrm{~N})$ & WPW & & $\mathrm{N}$ & $\mathrm{RD}$ & $\mathrm{N}$ & $\mathrm{N}$ & $\mathrm{M} / \mathrm{wt}$ \\
\hline $1 \| 1: 3$ & $M$ & 23 & $\mathrm{HCM}$ & 34 & 30 & 90 & 41 & 747 & WPW & 29 & 24 & & + & + & \\
\hline III:4 & $\mathrm{F}$ & 32 & $\mathrm{~N}$ & 47 & 8 & 65 & 34 & & WPW & & $\mathrm{N}$ & & $\mathrm{N}$ & $\mathrm{N}$ & $\mathrm{M} / \mathrm{wt}$ \\
\hline |II: $5^{*}$ & $M$ & 19 & $\overline{D C M}$ & 74 & & & 12 & $(\uparrow)$ & & 23 & 20 & & & & \\
\hline $\mid I I: 7^{*}$ & $M$ & 18 & DCM & 78 & & & 10 & $(\uparrow)$ & & 21 & & & & & \\
\hline $\mid I I: 9^{*}$ & $M$ & 22 & DCM & 72 & & & 14 & $(\uparrow)$ & & & 23 & & & & \\
\hline III:10 & $M$ & 13 & $\mathrm{HCM}$ & & & 40 & & 730 & WPW & & 22 & VD & + & + & $\bar{M}$ \\
\hline IV:1 & $M$ & & $\mathrm{HCM}$ & 43 & 11 & 54 & & 776 & WPW & & 23 & $\mathrm{RD}$ & + & + & $M$ \\
\hline IV:2 & $\mathrm{F}$ & 23 & $\mathrm{~N}$ & 49 & 11 & 69 & 43 & $(\mathrm{~N})$ & WPW & & $\mathrm{N}$ & RD & $\mathrm{N}$ & $\mathrm{N}$ & $\mathrm{M} / \mathrm{wt}$ \\
\hline IV-4 & $F$ & 13 & $\mathrm{~N}$ & 40 & 7 & 80 & 36 & $(\mathrm{~N})$ & WPW & & $\mathrm{N}$ & $\mathrm{RD}$ & $\mathrm{N}$ & $\mathrm{N}$ & $\mathrm{M} / \mathrm{wt}$ \\
\hline IV-5 & $F$ & 15 & $\mathrm{~N}$ & 44 & 9 & 80 & 31 & $(\mathrm{~N})$ & $\mathrm{N}$ & & $\mathrm{N}$ & RD & $\mathrm{N}$ & $\mathrm{N}$ & $\mathrm{M} / \mathrm{wt}$ \\
\hline IV:7 & $M$ & 14 & $\mathrm{HCM}$ & 56 & 19 & 27 & 17 & & WPW & & 19 & & + & + & $M$ \\
\hline $\mathrm{V}: 2$ & $M$ & 1 & $\mathrm{~N}$ & 34 & 9.5 & 79 & 41 & 346 & $\mathrm{~N}$ & & $\mathrm{~N}$ & & + & + & $M$ \\
\hline
\end{tabular}

Shaded cells indicate data not collected/available (in case of ocular involvement, indicates no exam done; in case of genotype, indicates no DNA was available)

${ }^{a}$ Data of deceased individuals reported by Towbin et al. (1993)

$D C M$ dilated cardiomyopathy; HCM hypertrophic cardiomyopathy; LVEDD left ventricular end diastolic dimension; IVSD Intraventricular septal diameter diastole; $E F$ ejection fraction; FS fractional shortening; $M M-C K$ creatine kinase level, muscle isoform (compared to upper limit of normal value); + present; $N$ negative or normal; $\uparrow$ elevated; $A F$ atrial fibrillation; $W P W$ Wolff Parkinson White; $I C D$ implantable cardiac defibrillator; $R D$ retinal disease; $V D$ visual deficit reported by patient; $M$ mutation carrier; $w t$ wild type allele

as a presenting feature in males is under-appreciated. In the present study, we confirm the diagnosis of Danon disease in the original XLCM-2 family, described the broad phenotypic manifestations within the expanded XLCM-2 pedigree, and highlight the importance of a DCM phenotype as a presenting sign in Danon disease.

\section{Patients and methods}

Since the original report of XLCM-2, two additional generations of relatives were available for study. Data from the original report (Towbin et al. 1993), as well as records obtained from our research subjects were collected under an approved human subjects protocol, providing clinical information on 16 family members (Table 1). Living relatives seen at our center underwent the following evaluations: medical history, physical examination, measurement of serum creatinine kinase, electrocardiogram, and echocardiograms (M-mode, 2D and color doppler), according to standard guidelines (Mestroni et al. 1999). Mutations in $D M D$ were excluded by clinical testing (City of Hope, Duarte, CA, USA) and we also excluded common mitochondrial mutations (Institute for Molecular and Human Genetics Molecular Diagnostic Laboratory, Georgetown, WA, USA). Polymerase chain amplification of the nine $L A M P-2$ exons was performed using the primers reported by Nishino et al. (2000), and DNA sequencing was performed on an ABI 377 DNA Sequencer (Applied Biosystem, Foster City, CA, USA). A collection of 150 ethnically similar healthy subjects were screened by pyrosequencing (PSQ 96MA, Biotage, Foxboro, MA, USA; primers and conditions available upon request) to exclude the possibility of our detected mutation being a common polymorphism. Skeletal muscle biopsy samples from one young male (V:2; Fig. 1) and from the explanted heart of an affected teenager (IV:7) were studied. Formalin fixed muscle specimens were stained by hematoxylin and eosin and trichrome, and were studied by immunohistochemsitry for the presence of LAMP-2. Immunostaining for LAMP-2 was performed on skeletal muscle exposed for 60 min to a 1:50 dilution of the H4B4 anti-human LAMP-2 antibody (Developmental Studies Hybridoma Bank, Iowa City, IA, USA) similar to previous descriptions (Fanin et al. 2006). Electron microscopy was performed on both specimens. 

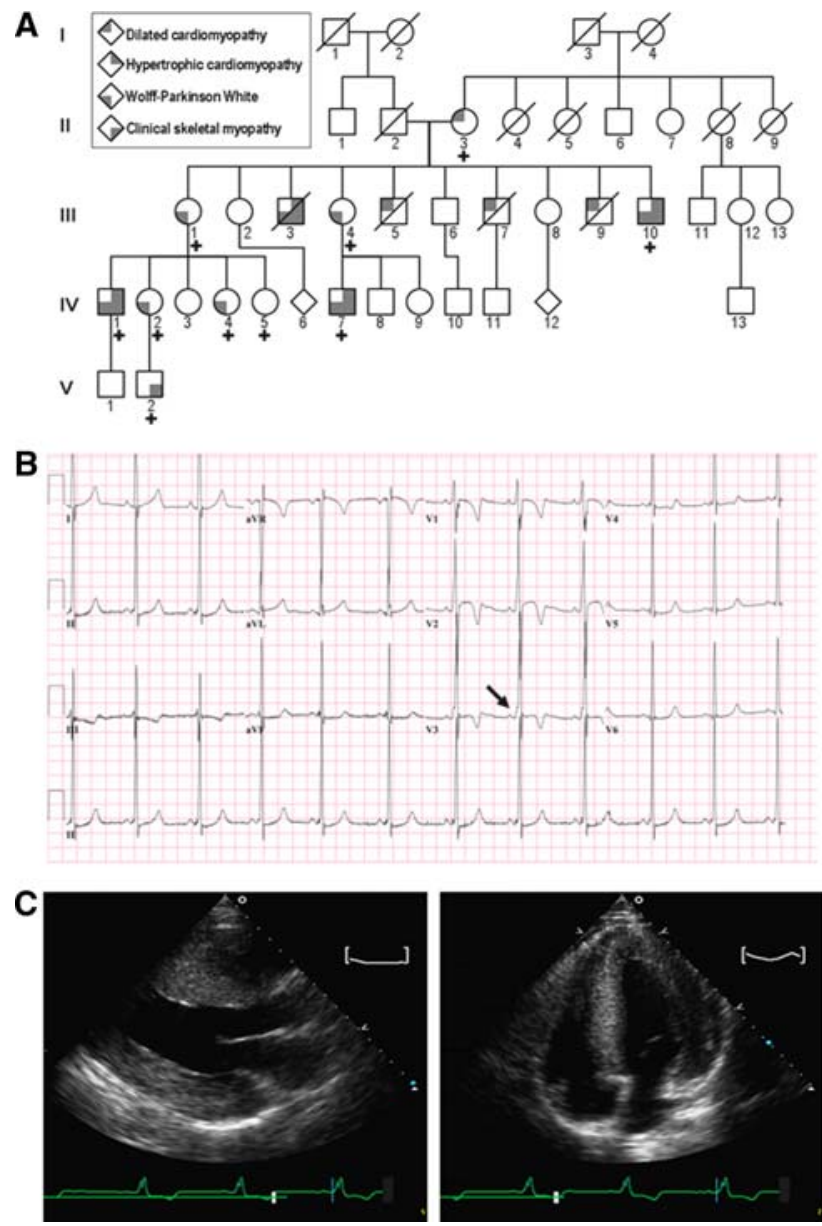

Fig. 1 Clinical features of family HCM-004 (XLCM-2). a Males and females are depicted by squares and circles, respectively and the phenotypes are indicated by shading. Individuals with known LAMP2 mutation are indicated with a plus sign (+) under their ID number; diagonal lines indicated deceased individuals and diamonds indicate more than one offspring. b Electrocardiogram tracing of a female (IV4) carrying a LAMP-2 mutation, demonstrating a short PR interval, delta waves and prominent voltage. $\mathbf{c}$ Echocardiogram images of male carrier (IV-7). The parasternal and four-chamber views show concentric hypertrophy involving septum, lateral and posterior left ventricular walls (diastolic left ventricular posterior wall and septum thickness 1.9 and 1.8 , respectively) associated with severe systolic dysfunction (left ventricular ejection fraction 27\%)

\section{Results}

\section{Clinical findings}

Clinical phenotypes for this family are summarized in Table 1. The presenting phenotype in the family was DCM, present in the female proband (II:3) and three of her sons (III:5, III:7, III:9). One male (III:3) is listed in the original study as having DCM, although records available on this individual later supported a HCM phenotype
(Towbin et al. 1993). The DCM was more severe in males who had severe dilatation and systolic dysfunction; all succumbed to their disease in the 3rd decade and surviving records in three showed elevated creatinine kinase levels and skeletal muscle symptoms in one. Subsequent to the original report, three other male relatives (III:10, IV:1, IV:7) developed severe concentric HCM (Figs. 1, 3) associated with Wolff-Parkinson-White syndrome (WPW). These males also had a severe prognosis, with progression to intractable heart failure, and death or transplant occurring between 15 and 24 years. Three adult women had WPW as their only cardiac manifestation. Affected males had elevated creatinine kinase, skeletal muscle weakness, and reported mild cognitive impairment. In contrast to previous reports, carrier females in this family had non-cardiac and non-skeletal muscle manifestations of disease, including retinal pigmentary changes (III:1 and IV:2) and transient visual loss. On retinal exam of four females and one male carrying the mutation, abnormalities of the retinal pigment epithelium were found; this included retinal disease in two women who had no sign of cardiac disease at the time of retinal exam. Details of the retinal findings in this family and in another unrelated patient have recently been published (Prall et al. 2006).

Sequence analysis of $L A M P-2$ revealed a novel hemizygous (males) or heterozygous (females) single nucleotide deletion in exon 8 (NM_002294.1:c.delA1219) predicting a frameshift affecting codon 361 (NP_002286:pof length 410 amino acids) and the addition of 18 or 15 novel amino acids to the LAMP-2A (NP_002286:pGln361SerfsX18) and LAMP-2B (NP_002286:pGln361SerfsX15) isoform of the LAMP-2 protein respectively. The mutation obliterates the carboxy sequences for both the lysosomal transmembrane and lysosomal targeting domains, predicting that the protein, if produced, would be unable to target to the lysosome or integrate into the lysosomal membrane (Nishino et al. 2000) Native LAMP-2 protein was absent by Western blotting in lymphocytes and cardiac muscle tissue from affected males (data not shown). The mutation was present in all affected individuals and in obligate carriers tested. The mutation was absent in over 300 control chromosomes, and the predicted novel amino acid sequences distal to the frameshift mutation have no known similarity with other described proteins or domains. Review of a skeletal biopsy of a 13-month-old male who carries the mutation and presented with muscle weakness $(\mathrm{V}: 2)$ showed only nonspecific findings and absence of LAMP-2 protein; but evidence of abnormal vacuolization was seen on electron microscopy (Fig. 2). Standard immunostaining for dystrophin, merosin, and saroglycan proteins was normal. A report of individual III:3's muscle biopsy noted "multiple vacuoles that occur randomly 
Fig. 2 Skeletal muscle specimen from patient $\mathrm{V}: 2$ at age 13 months. a

Photomicrograph (hematoxylin and eosin stain) of quadriceps muscle biopsy showing nonspecific changes and absence of demonstrable vacuolization (Bar $100 \mu \mathrm{m})$. b Electron micrograph demonstrating occasional presence of autophagic vacuoles characteristic of Danon disease (Bar $1 \mu \mathrm{m})$. c Photomicrograph showing immunohistochemical reactivity for $L A M P-2$ in lysosomes (arrow) of positive control specimen (Bar $100 \mu \mathrm{m})$. d Photomicrograph showing absence of reactivity for LAMP2 in patient V:2 (Bar $100 \mu \mathrm{m})$. Note: $\mathbf{c}$ and $\mathbf{d}$ represent formalin-fixed muscle tissues

Fig. 3 Cardiac muscle specimen from patient IV:7 at age 14 years. a Cross section of explanted heart showing biventricular hypertrophy and fibrosis. b Photomicrograph (trichrome stain) showing the extensive fibrosis in blue and the cardiomyocytes in red (Bar $100 \mu \mathrm{m})$. c Photomicrograph (hematoxylin and eosin stain) of showing extensive vacuolization of cardiomyocytes (Bar $100 \mu \mathrm{m}$ ). d Electron micrograph showing multiple autophagic vacuoles (arrows), correlating with the cytoplasmic vacuolization observed by light microscopy (Bar $1 \mu \mathrm{m})$
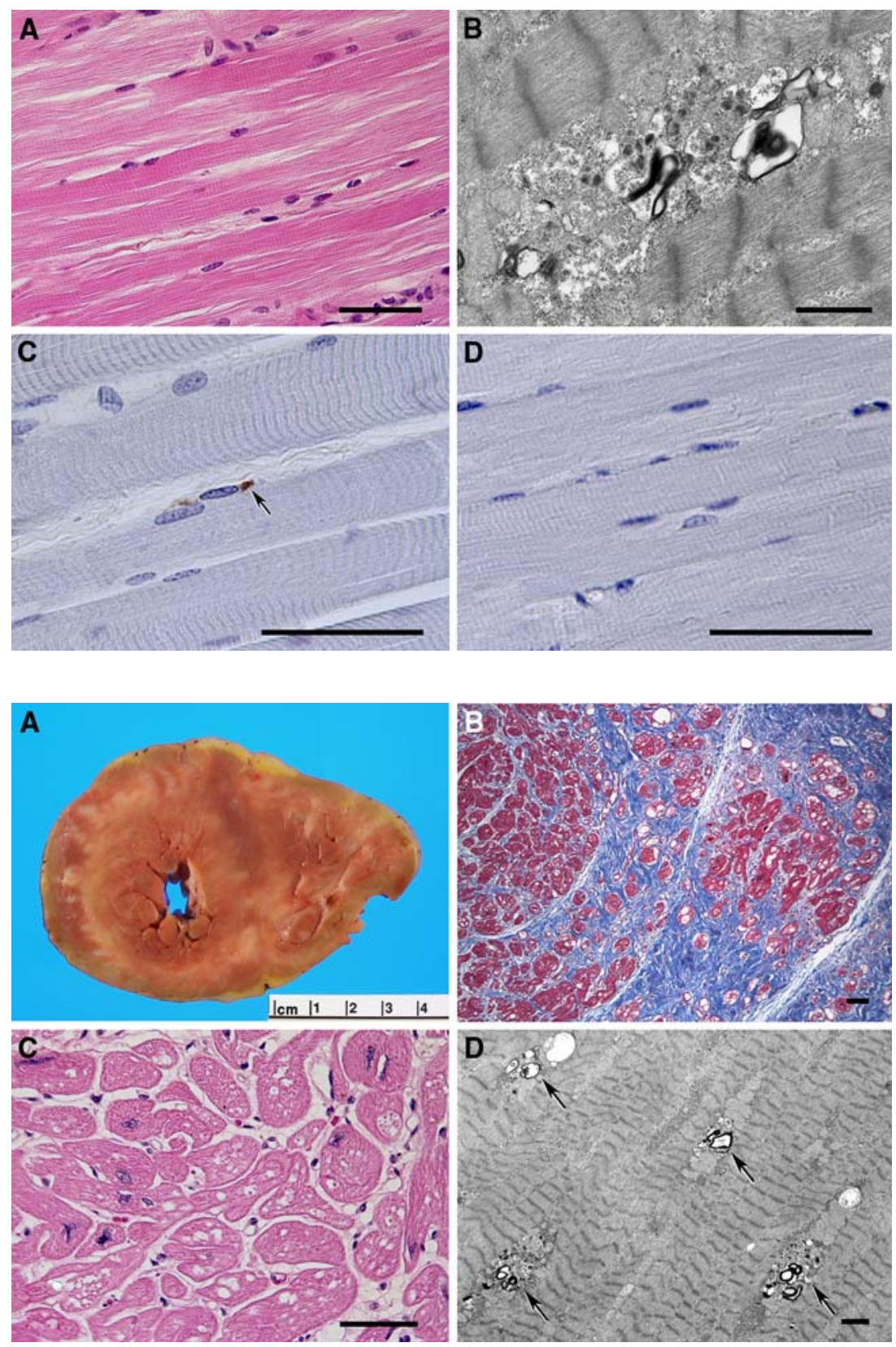

throughout the fiber and prominent autophagocytic vacuoles that are lined by a single membrane". Cardiac histology from individual IV:7 showed severe myocyte hypertrophy and the expected pattern of prominent vacuolization in the myocardium (Fig. 3).

\section{Discussion}

In this report we provide a molecular diagnosis for one of the original XLCM families, confirming a diagnosis of Danon disease instead of a dystrophinopathy as was initially suspected. Four presenting males in this family had
DCM (Towbin et al. 1993) instead of the "expected" HCM that has been typically described in males with Danon disease. Although DCM has been described in conjunction with HCM in Danon disease, males are typically described as having HCM, and we believe our family to be the first to present with a predominant severe DCM in males. Three recent studies have drawn attention to $\mathrm{HCM}$ as presenting feature of LAMP-2 mutations, pointing out the importance of the recognition of this disorder (Yang et al. 2005; Charron et al. 2004; Arad et al. 2005). The DCM phenotype in males has been less widely described and may account for roughly $10 \%$ of cases (Sugie et al. 2002). The prevalence of $L A M P-2$ mutations in idiopathic DCM is 
presently unknown and presents a diagnostic challenge, as for Danon disease males this represents an "uncommon" presentation of a disease unfamiliar to most physicians.

A broad range of cardiac and extra-cardiac phenotypes were present in XLCM-2. The presence of pre-excitation as the sole cardiac manifestation in four women in XLCM-2 is distinct from prior reports of Danon disease where women also had overt cardiomyopathy (Sugie et al 2002). WPW appears to be a feature of "metabolic" cardiomyopathies, as it has also been described for Pompe and cardiomyopathy due to AMP-activated protein kinase gene (PRKAG2) mutations (Francesconi et al. 1982; Francesconi and Auff 1982; Bulkley and Hutchins 1978; Murphy et al. 2005). WPW appears rare in cardiomyopathies due to mutations in cytoskeletal or sarcomeric genes. The origin of pre-excitation in Danon disease is unknown, but the analogy with PRKAG2 may implicate disruption of the annulus fibrosus by glycogen-engorged myocytes, as observed in mouse models (Arad et al. 2003).

Females did not exhibit overt muscular dystrophy signs, although one female reported fatigue and had a single elevated creatine kinase level that returned to normal on repeat testing. The muscle biopsy performed in the 13-month-old male was nonspecific, and implies that light microscopic studies of early muscle biopsy may lack diagnostic sensitivity, presumably because prominent accumulation of vacuoles has not yet occurred. In one other report, a progression of vacuolization changes was described in a male who underwent biopsy at ages 20 months and 16 years (Sugie et al. 2003). The data on human cardiac biopsies in Danon disease are more limited, although severe vacuolization changes as reported here and by others mirror the skeletal muscle findings (Nishino et al. 2000; Arad et al. 2005; Lacoste-Collin et al. 2002). In this study, electron microscopy proved to be a more sensitive technique in the young male's muscle biopsy, enabling easy detection and confident identification of the relatively few inclusions that were already present. The absence of $L A M P-2$ reactivity, demonstrated by immunohistochemical staining, provided confirmatory evidence that these represented aberrant autophagosomes. Learning difficulties were present in affected males, and included difficulties performing in school, particularly with mathematics; frank mental retardation with IQ levels below 70 was not found. The retinal disease in this family preceded overt cardiac disease in women, and may be a specific early clinical clue to Danon disease (Prall et al. 2006). The phenotype of mice hemizygous for LAMP-2 knockout mutations has not been welldescribed, and it remains unclear why hemizygous human females should exhibit these retinal changes without other significant signs of central nervous system involvement.

In conclusion, we have provided a molecular diagnosis for the originally reported XLCM-2 family. In contrast to other reports of Danon disease, the presenting phenotype in males was a severe DCM, with the more typical HCM phenotype manifesting in the more recent generations of the family. In women, WPW was a prominent feature, but extra-cardiac manifestations were also reported. The nonspecific muscle biopsy findings in a young male attest to the limited sensitivity of light microscopy in diagnosing Danon disease in young patients. Finally, in patients with unexplained DCM that could be due to X-linked inheritance, molecular genetic testing for LAMP-2 mutations should be considered, especially if the family history suggests skeletal muscle disease, WPW, cognitive delays, or HCM.

Acknowledgments We acknowledge the contributions of other participating scientists of the Familial Cardiomyopathy Registry: Andrea Di Lenarda, Gianfranco Sinagra, Snjezana Miocic, Francesca Brun, Gregory Schwartz, Carlin Long, William Baker, Bertron Groves, Deborah Ferguson, Cathleen Kenny, Arlene Drack, and Jeffrey Olson. Also acknowledged are Dr. Mark Haimann and the participating patients and their families.

\section{References}

Arad M, Moskowitz IP, Patel VV et al (2003) Transgenic mice overexpressing mutant PRKAG2 define the cause of WolffParkinson-White syndrome in glycogen storage cardiomyopathy. Circulation 107:2850-2856

Arad M, Maron BJ, Gorham JM et al (2005) Glycogen storage diseases presenting as hypertrophic cardiomyopathy. N Engl J Med 352:362-372

Berko BA, Swift M (1987) X-linked dilated cardiomyopathy. N Engl J Med 316:1186-1191

Bulkley BH, Hutchins GM (1978) Pompe's disease presenting as hypertrophic myocardiopathy with Wolff-Parkinson-White syndrome. Am Heart J 96:246-252

Charron P, Villard E, Sebillon P et al (2004) Danon's disease as a cause of hypertrophic cardiomyopathy: a systematic survey. Heart 90:842-846

Danon MJ, Oh SJ, DiMauro S et al (1981) Lysosomal glycogen storage disease with normal acid maltase. Neurology 31:51-57

Fanin M, Nascimbeni AC, Fulizio L, Spinazzi M, Melacini P, Angelini C (2006) Generalized lysosome-associated membrane protein-2 defect explains multisystem clinical involvement and allows leukocyte diagnostic screening in Danon disease. Am J Pathol 168:1309-1320

Francesconi M, Auff E (1982) Cardiac arrhythmias and the adult form of type II glycogenosis. N Engl J Med 306:937-938

Francesconi M, Auff E, Ursin C, Sluga E (1982) [WPW syndrome combined with AV block 2 in an adult with glycogenosis (Type II)]. Wien Klin Wochenschr 94:401-404

Lacoste-Collin L, Garcia V, Uro-Coste E et al (2002) Danon's disease (X-linked vacuolar cardiomyopathy and myopathy): a case with a novel Lamp-2 gene mutation. Neuromuscul Disord 12:882-885

Mestroni L, Maisch B, McKenna WJ et al (1999) Guidelines for the study of familial dilated cardiomyopathies. Collaborative Research Group of the European Human and Capital Mobility Project on Familial Dilated Cardiomyopathy. Eur Heart J 20: 93-102

Murphy RT, Mogensen J, McGarry K et al (2005) Adenosine monophosphate-activated protein kinase disease mimicks 
hypertrophic cardiomyopathy and Wolff-Parkinson-White syndrome: natural history. J Am Coll Cardiol 45:922-930

Nishino I, Fu J, Tanji K et al (2000) Primary LAMP-2 deficiency causes X-linked vacuolar cardiomyopathy and myopathy (Danon disease). Nature 406:906-910

Ortiz-Lopez R, Li H, Su J, Goytia V, Towbin JA (1997) Evidence for a dystrophin missense mutation as a cause of X-linked dilated cardiomyopathy. Circulation 95:2434-2440

Prall FR, Drack A, Taylor M et al (2006) Ophthalmic manifestations of Danon disease. Ophthalmology 113:1010-1013

Sugie K, Yamamoto A, Murayama K et al (2002) Clinicopathological features of genetically confirmed Danon disease. Neurology 58:1773-1778
Sugie K, Koori T, Yamamoto A et al (2003) Characterization of Danon disease in a male patient and his affected mother. Neuromuscul Disord 13:708-711

Towbin JA, Hejtmancik F, Brink P et al (1993) X-linked cardiomyopathy (XLCM): molecular genetic evidence of linkage to the Duchenne muscular dystrophy (dystrophin) gene at the Xp21 locus. Circulation 87:1854-1865

Yang Z, McMahon CJ, Smith LR et al (2005) Danon disease as an underrecognized cause of hypertrophic cardiomyopathy in children. Circulation 112:1612-1617 